EPJ manuscript No.

(will be inserted by the editor)

\title{
Scale free effects in world currency exchange network
}

\author{
A. Z. Górski ${ }^{1}$, S. Drożdż $\dot{z}^{1,2}$, and J. Kwapień ${ }^{1}$ \\ ' 1 Institute of Nuclear Physics, Polish Academy of Science, PL-31-342 Kraków, Poland \\ 2 Institute of Physics, University of Rzeszów, PL-35-310 Rzeszów, Poland \\ Received: date / Revised version: date
}

\begin{abstract}
A large collection of daily time series for 60 world currencies' exchange rates is considered. The correlation matrices are calculated and the corresponding Minimal Spanning Tree (MST) graphs are constructed for each of those currencies used as reference for the remaining ones. It is shown that multiplicity of the MST graphs' nodes to a good approximation develops a power like, scale free distribution with the scaling exponent similar as for several other complex systems studied so far. Furthermore, quantitative arguments in favor of the hierarchical organization of the world currency exchange network are provided by relating the structure of the above MST graphs and their scaling exponents to those that are derived from an exactly solvable hierarchical network model. A special status of the USD during the period considered can be attributed to some departures of the MST features, when this currency (or some other tied to it) is used as reference, from characteristics typical to such a hierarchical clustering of nodes towards those that correspond to the random graphs. Even though in general the basic structure of the MST is robust with respect to changing the reference currency some trace of a systematic transition from somewhat dispersed - like the USD case - towards more compact MST topology can be observed when correlations increase.
\end{abstract}

PACS. 89.65.Gh Economics; econophysics, financial markets, business and management - 89.75.Fb Structures and organization in complex systems - 05.45.Tp Time series analysis

The world currency exchange market (foreign exchange - FOREX, FX) is the world largest financial market and it constitutes an extremely complex network. The FX daily takeover volume is of the order of $10^{12}$ USD. Any other financial market can hardly approach such volume. Also, this market has direct influence on all other markets because any price is expressed in terms of a currency. The large volume makes it virtually impossible to control from outside and there is no friction (transactions are basically commission free). Due to time differences FX transactions are performed 24 hours a day, 5.5 day a week with maximum volume between 1 and 4 p.m. GMT, when both American and European markets are open. Hence, the FX time series relations represent an exceptionally complex network indeed, and they are therefore especially worth of detailed analysis. The FX market can be viewed as a complex network of mutually interacting nodes, each node being an exchange rate of two currencies. In principle, all nodes are interconnected with complex nonlinear interactions. Any currency can be expressed in terms of particular one that is called the base currency. In spite of its importance, much less attention has been paid in literature to the FX cross-correlation analysis, than to such analysis of stock markets 1,2,3. Therefore in the following the currency network will be analyzed. Our motivation to investigate correlations of FX time series is twofold: theoretical and practical. More detailed correlation anal- ysis can give insight into the structure of links between various currencies and, in particular, it potentially may provide quantitative arguments in favor of an often postulated hierarchical organization of world currency exchange market. Knowledge of correlations is also essential for the portfolio management.

Usually, for a financial time series of an ith asset $(i=$ $1, \ldots, n)$ at time $t, x_{i}(t)=x_{i}$, one defines its return over time period $\tau$ as $G_{i}(t ; \tau)=\ln x_{i}(t+\tau)-\ln x_{i}(t)$. For FX series instead of a value $x_{i}(t)$ one has $x_{A}^{B}(t)$, an exchange rate, i.e. a value of currency $A$ expressed in terms of a base currency $B$. Hence, the returns can be denoted as $G_{A}^{B}(t)$ and they are clearly antisymmetric $G_{A}^{B}(t ; \tau)=$ $-G_{B}^{A}(t ; \tau)$. FX returns, due to the lack of commission and high liquidity satisfy the triangle rule: $G_{A}^{B}(t ; \tau)+$ $G_{B}^{C}(t ; \tau)+G_{C}^{A}(t ; \tau)=0$, already for relatively small values of $\tau$. As a result, for a set of $n$ currencies we have $N=n-1$ independent values and the same number of nodes with a given base currency.

In the following we analyze time series of daily data for 60 currencies, including gold, silver and platinum [5]. The data taken covers the time period Dec 1998-May 2005. In order to automatically get rid of possible misprints in the original data the daily jumps greater than $5 \sigma$ (less than $0.3 \%$ of data points) were removed. Also, the gaps related to non-trading days were synchronized. For each exchange rate we thus obtain a time series of 1657 data points. The 
currencies are denoted according to ISO 4217 standard, and they can be formally divided into four groups, according to their liquidity. The major currencies, that we call the $A^{\star}$ group, include USD, EUR, JPY, GBP, CHF, CAD, AUD, NZD, SEK, NOK, DKK (11 currencies). All other liquid currencies belong to the group $A$ (CYP, CZK, HKD, HUF, IDR, ILS, ISK, KRW, MXN, MYR, PHP, PLN, SGD, SKK, THB, TRY, TWD, XAG, XAU, XPT, ZAR, 21 currencies). Less liquid currencies (group $B$ ) include: ARS, BGN, BRL, CLP, KWD, RON, RUB, SAR, TTD (9 currencies). Finally, the non-tradable currencies (group $C$ ) taken into account are: AED, COP, DZD, EGP, FJD, GHC, HNL, INR, JMD, JOD, LBP, LKR, MAD, PEN, PKR, SDD, TND, VEB, ZMK (19 currencies). In the latter group the exchange rates are usually fixed daily by national central banks. Dividing currencies into such four groups of different liquidity is common among finance practitioners. This therefore opens an additional interesting issue to be verified if the different dynamics that stays behind such a division according to the liquidity (implying the fixing method) is also reflected in correlations of daily exchange rates.

For a given choice of the base currency $X$ the (symmetric) correlation matrix $(\mathrm{CM})$ can be computed in terms of the normalized returns, $g_{A}^{X}(t)$. To this end one takes $N$ time series $\left\{g_{A}^{X}\left(t_{0}\right), g_{A}^{X}\left(t_{0}+\tau\right), \ldots, g_{A}^{X}\left(t_{0}+(T-1) \tau\right)\right\}$ of length $T$. These series can form an $N \times T$ rectangular matrix $\boldsymbol{M}^{X}$, and the $\mathrm{CM}$ can be written in the matrix notation as

$$
\boldsymbol{C}^{X} \equiv\left[C^{X}\right]_{A B}=\frac{1}{T} \boldsymbol{M}^{X} \widetilde{\boldsymbol{M}}^{X}
$$

where tilde stands for the matrix transposition. By construction the trace of the $\mathrm{CM}$ is equal to the number of time series $\operatorname{Tr} C^{X}=N$. When some of the time series become strongly dependent, the related number of eigenvalues approach value zero (zero modes). This effect, typical for FX data, does not occur for the stock market time series.

Sample spectra of CMs for the same time series as the ones considered here, using several different base currencies $X$, can be found in 6 . In all the cases one finds a maximal eigenvalue $\left(\lambda_{\max }\right.$, in our case $\left.\max =N=59\right)$ that is well separated from all the remaining eigenvalues and is placed within the range $0.2 N \div 0.9 N$, with average around $0.5 N$. With a few exceptions, there are also clearly isolated second maximal eigenvalues $\left(\lambda_{\max -1}\right)$, considerably smaller than $\lambda_{\max }$, but also well separated from the bulk of other eigenvalues. At the same time, the magnitude of the largest eigenvalue significantly depends on which currency is used as a base $X$. The largest maximal eigenvalues correspond to those base currencies that either have a very strong drift, independent of the behavior of other currencies (like GHC) or whose fluctuations are to a large extent independent of the global FX behavior. The smallest values of $\lambda_{\max }$ appear when the USD or other currencies that are strongly tied to it are used as $X$. This reflects the fact that changes in value of the USD - due to its global world significance - cause a rich diversity of reactions of all other currencies. In other words, their dy- namics viewed from the USD perspective looks less cooperative as compared to the cases when the base currency is not that influential. Thus, effectively eliminating the USD by using it as the base currency sizeably diminishes correlations among all other currencies. Another interesting manifestation of this effect will be seen in the Minimal Spanning Tree (MST) graphs picture. It should be mentioned that analogous characteristics for eigenvalues have been identified in the smaller subsectors (like tradable and non-tradable separately) of the analyzed currencies.

The structure of eigenspectrum is one way to get insight into large amount of data as represented by the correlation matrices. A complementary - efficient and conclusive - way to visualize such ensembles of data is in terms of the MST graphs. In the following we use the MST graphs approach in order to further explore properties of the currency exchange network. The MST was introduced in graph theory quite long ago 7,8. Later it was rediscovered several times 9,10 . To analyze the stock market correlations it was applied by Mantegna [11] and later on by several authors [12,13,16,17. Recently MST graphs were used to study FX correlations [18, 19,20]. Here we present the most exhaustive results concerning the FX data, for 60 world currencies.

To construct the MST graph we choose the following measure

$$
d^{X}(A, B)=\sqrt{\left(1-C_{A B}^{X}\right) / 2}, \quad d^{X}(A, B) \in[0,1],
$$

that satisfies the standard axioms of metric. The distance $d$ between two time series is smaller if their correlation coefficient is closer to unity. One node (vertex) in the graph corresponds to each time series. We shall connect the two nodes, $A$ and $B$, with a line (edge, "leg"), if $d(A, B)$ is the smallest. In the next step we look for another two closest nodes and again we connect them with a line. This procedure is repeated until we obtain a connected tree graph.

We call multiplicity (degree) of that node the number of legs attached to it. Clearly, multiplicity of a node is an integer number, $K$. In our case we have $N=59$ nodes for a given base currency. As examples, Fig. 1 shows the MST graphs for our set of data for three major currencies, USD, GBP and JPY, taken as the base currency. For a node $A$ its multiplicity is denoted by $K_{A}$. Integer $N^{\prime}(K)$ is the number of nodes with exactly $K$ legs. Because the total number of nodes, $N$, is relatively small, we introduce the integrated quantity

$$
F(K)=N(K) / N=\frac{1}{N} \sum_{I=K}^{K_{\max }} N^{\prime}(I),
$$

where $K_{\max }$ denotes the maximal number of legs in the MST graph. $N(K)$ is the number of nodes with $K$ or more legs. Counting legs in all nodes of our MST graph we can construct discrete function $N(K)$ and its normalized version, $F(K)$.

In Fig. 2 we show the $F(K)$ plots for the MST node's multiplicity for different base currencies from each of the four groups, $A, A^{\star}, B$ and $C$. To make the plots readable 

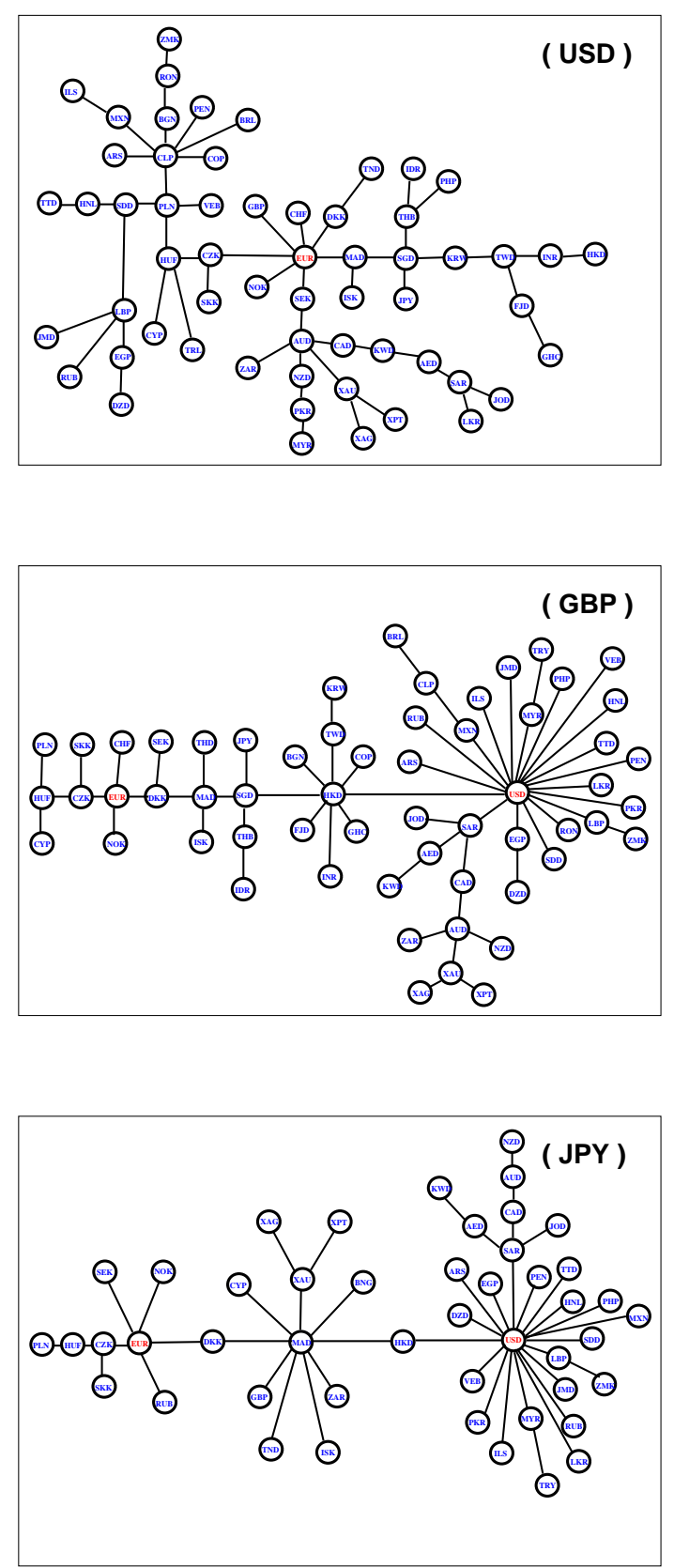

Fig. 1. MST graphs for all the currencies taking USD, GBP and JPY as the base currency, respectively.

we present the distributions for five typical basic currencies from each group. Already at first glimpse, basically all plots indicate scaling and their similarity extends even to a quantitative level. One subtle difference can be visible when the USD (as well as the other currencies linked to it) is used as reference. Here, in the log-log scale one observes a somewhat faster decrease than linear. Nevertheless, fitting it with the straight line results thus in a steeper slope and the worse quality of such a fit. The relevant detailed numerical parameters, including the power exponent $\alpha$, its standard error $(\Delta \alpha)$, and values of the maximal CM eigenvalues, for all the 60 currencies used as the base for the remaining ones are listed in Tables 1-4. The exponents
Table 1. Characteristics of the power fits for the MST node's multiplicity of all the currencies using the base currencies from the group $A^{*}$.

\begin{tabular}{|ccccc|}
\hline \hline base currency & $\alpha$ & $\Delta \alpha$ & $\Delta \alpha / \alpha$ & $\lambda_{\max }$ \\
\hline \hline AUD & 1.43 & 0.08 & $5.4 \%$ & 32.1 \\
CAD & 1.39 & 0.11 & $7.7 \%$ & 23.2 \\
CHF & 1.34 & 0.08 & $6.0 \%$ & 29.9 \\
DKK & 1.33 & 0.08 & $5.9 \%$ & 27.4 \\
EUR & 1.30 & 0.08 & $5.9 \%$ & 27.6 \\
GBP & 1.44 & 0.11 & $7.6 \%$ & 24.1 \\
JPY & 1.36 & 0.08 & $6.0 \%$ & 32.6 \\
NOK & 1.41 & 0.08 & $5.5 \%$ & 28.4 \\
NZD & 1.43 & 0.07 & $5.0 \%$ & 34.4 \\
SEK & 1.41 & 0.07 & $4.7 \%$ & 29.2 \\
USD & 1.92 & 0.18 & $9.6 \%$ & 11.8 \\
\hline average & 1.43 & 0.09 & $6.3 \%$ & 27.3 \\
\hline \hline
\end{tabular}

Table 2. Characteristics of the power fits for the MST node's multiplicity of all the currencies using the base currencies from the group $A$.

\begin{tabular}{|ccccc|}
\hline \hline base currency & $\alpha$ & $\Delta \alpha$ & $\Delta \alpha / \alpha$ & $\lambda_{\max }$ \\
\hline \hline CYP & 1.42 & 0.07 & $4.6 \%$ & 36.6 \\
CZK & 1.33 & 0.08 & $5.8 \%$ & 31.7 \\
HKD & 2.31 & 0.32 & $14.0 \%$ & 11.7 \\
HUF & 1.38 & 0.08 & $5.8 \%$ & 31.8 \\
IDR & 1.48 & 0.09 & $5.9 \%$ & 43.7 \\
ILS & 1.56 & 0.12 & $7.9 \%$ & 24.2 \\
ISK & 1.47 & 0.09 & $5.9 \%$ & 27.6 \\
KRW & 1.59 & 0.11 & $6.7 \%$ & 27.8 \\
MXN & 1.51 & 0.07 & $4.3 \%$ & 29.8 \\
MYR & 2.25 & 0.31 & $14.0 \%$ & 11.9 \\
PHP & 1.62 & 0.11 & $6.7 \%$ & 24.5 \\
PLN & 1.49 & 0.08 & $5.7 \%$ & 32.0 \\
SGD & 1.67 & 0.10 & $5.9 \%$ & 13.8 \\
SKK & 1.41 & 0.06 & $4.4 \%$ & 31.7 \\
THB & 1.50 & 0.11 & $7.3 \%$ & 20.5 \\
TRY & 1.55 & 0.08 & $5.1 \%$ & 43.3 \\
TWD & 1.61 & 0.12 & $7.7 \%$ & 15.2 \\
XAG & 1.48 & 0.11 & $7.7 \%$ & 46.7 \\
XAU & 1.38 & 0.08 & $5.5 \%$ & 37.2 \\
XPT & 1.40 & 0.09 & $6.3 \%$ & 47.1 \\
ZAR & 1.50 & 0.08 & $5.7 \%$ & 41.8 \\
\hline average & 1.57 & 0.11 & $6.8 \%$ & 30.0 \\
\hline \hline
\end{tabular}

$\alpha$ and their standard errors $\Delta \alpha$ were determined with the least square fit of corresponding power functions in the original linear scale. Because the total number of data points equals 59 and the number of occupied bins is typically around 10 more sophisticated statistical estimates do not seem adequate in this case.

It can be concluded that the scale free behavior and thus the power like fit are fairly good for majority of the base currencies. The worst scaling (if at all), $\Delta \alpha / \alpha \geq 9 \%$, was obtained for the USD and currencies tied to it (HKD, MYR, LBP). A technical reason for the latter effect is, that taking USD as the base currency one eliminates its 
A. Z. Górski et al.: Scale free effects in world currency exchange network
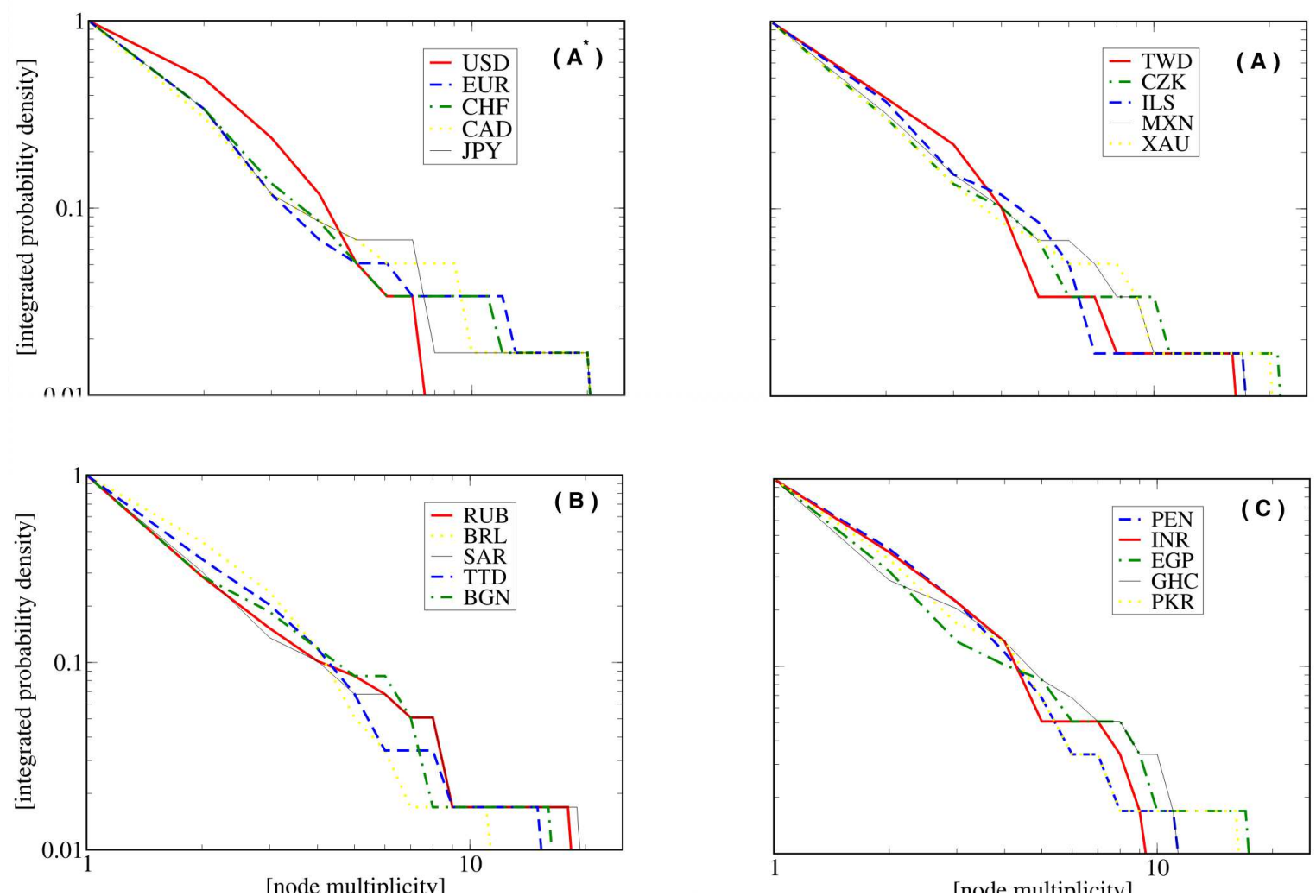

Fig. 2. Log-log plots of the function $F(K)$ for five sample base currencies taken from each group: $A^{*}, A, B$ and $C$, respectively.

Table 3. Characteristics of the power fits for the MST node's multiplicity of all the currencies using the basic currencies from the group $B$.

\begin{tabular}{|ccccc|}
\hline \hline base currency & $\alpha$ & $\Delta \alpha$ & $\Delta \alpha / \alpha$ & $\lambda_{\max }$ \\
\hline \hline AED & 1.92 & 0.13 & $6.5 \%$ & 17.5 \\
ARS & 1.45 & 0.06 & $4.4 \%$ & 40.2 \\
BGN & 1.44 & 0.09 & $5.3 \%$ & 32.3 \\
BRL & 1.60 & 0.11 & $6.7 \%$ & 43.5 \\
CLP & 1.48 & 0.09 & $6.0 \%$ & 31.0 \\
KWD & 1.85 & 0.11 & $6.1 \%$ & 17.9 \\
RON & 1.50 & 0.08 & $5.0 \%$ & 36.7 \\
RUB & 1.48 & 0.09 & $5.8 \%$ & 44.5 \\
SAR & 1.65 & 0.09 & $5.3 \%$ & 16.9 \\
TTD & 1.97 & 0.14 & $7.1 \%$ & 15.6 \\
\hline average & 1.63 & 0.09 & $5.8 \%$ & 29.6 \\
\hline \hline
\end{tabular}

node from the MST graph. Hence, the largest cluster (i.e. large multiplicity node) disappears, the tail of the distribution is getting thinner and the MST graph is too small to be able to reproduce fat tails (i.e. power like distribution). On a more fundamental level, this characteristic feature associated with the USD - as seen from the MST perspective - may reflect its special status in the world economy during the period considered here. It is probably also worth to notice that the quality of scaling goes
Table 4. Characteristics of the power fits for the MST node's multiplicity of all the currencies using the base currencies from the group $C$.

\begin{tabular}{|ccccc|}
\hline \hline base currency & $\alpha$ & $\Delta \alpha$ & $\Delta \alpha / \alpha$ & $\lambda_{\max }$ \\
\hline \hline COP & 1.46 & 0.07 & $7.1 \%$ & 30.8 \\
DZD & 1.44 & 0.08 & $6.3 \%$ & 51.3 \\
EGP & 1.50 & 0.32 & $4.7 \%$ & 39.5 \\
FJD & 1.48 & 0.08 & $6.0 \%$ & 31.0 \\
GHC & 1.54 & 0.09 & $4.9 \%$ & 52.2 \\
HNL & 1.81 & 0.12 & $5.0 \%$ & 14.0 \\
INR & 1.92 & 0.09 & $5.5 \%$ & 14.3 \\
JMD & 1.66 & 0.09 & $9.0 \%$ & 35.0 \\
JOD & 1.80 & 0.07 & $5.5 \%$ & 20.6 \\
LBP & 1.31 & 0.31 & $9.0 \%$ & 24.6 \\
LKR & 1.73 & 0.11 & $7.3 \%$ & 18.5 \\
MAD & 1.37 & 0.08 & $11.0 \%$ & 21.1 \\
PEN & 1.78 & 0.10 & $7.5 \%$ & 17.4 \\
PKR & 1.70 & 0.06 & $4.7 \%$ & 25.1 \\
SDD & 1.67 & 0.11 & $5.8 \%$ & 25.8 \\
TND & 1.39 & 0.08 & $8.0 \%$ & 21.6 \\
VEB & 1.44 & 0.12 & $5.8 \%$ & 37.6 \\
ZMK & 1.31 & 0.11 & $7.7 \%$ & 49.3 \\
\hline average & 1.57 & 0.11 & $6.7 \%$ & 29.4 \\
\hline \hline
\end{tabular}


somewhat in parallel with the magnitude of the largest eigenvalue (last column in the Tables) of the original correlation matrix. The disputable - as far as the scaling is concerned - cases are typically associated with the relatively small magnitudes of $\lambda_{\max }$ and thus with the lower degree of collectivity.

For the prevailing majority of the base currencies the statistical significance of the scaling effects is however quite convincing. Even more, the corresponding scaling exponents do not differ much among the four groups of currencies considered. The scale free behavior of MST graphs is in agreement with the successful usage of hierarchical structure methods in finance [20] and the fat tails are signature of currency clustering in MST graphs. In more quantitative terms, the power like scaling of nodes' degree has been shown in hierarchical networks [21,22 and the corresponding scaling exponents derived analytically. In the context of our present study it is very interesting and encouraging to find out that the average value we have obtained for the tail index is $\langle\alpha\rangle=1.55$ while the hierarchical model discussed in [21,22] implies very close value (for the cumulative distribution)

$$
\alpha_{\text {hier }}=\ln M / \ln (M-1)=1.61 .
$$

The replication factor $M$ to be used in this formula in our case can be calculated from the average node degree: $M=\langle K\rangle+1=2(N-1) / N+1=2.966$. The scaling exponent (4) thus differs from our average value $\langle\alpha\rangle$ by less than $4 \%$. This fact provides a significant quantitative argument in favor of the hierarchical organization of the world currency exchange market, an effect which already can visually be inferred from the panels corresponding to GBP and JPY in Fig. 1.

The panel corresponding to the USD looks less explicit in this respect. Indeed the quality of the corresponding scaling is here not so convincing. Nevertheless, approximation with the power like fit gives the exponent $\alpha$ which is significantly larger (Table 1) than in the above hierarchical model. In fact, the node's multiplicity distribution corresponding to this case and seen in Fig 1, develops some departure towards a Gaussian distribution and thus indicates some admixture of randomness and a more "democratic" multiplicity distribution as compared to a pure hierarchical situation. This effect also is consistent - as expressed by the corresponding $\lambda_{\max }$ in Table 1 - with the weakest synchronization of the currency exchange rates expressed in the USD. Interestingly, a correspondence of this kind seems to be leaving imprints for the other currencies when they are used as reference for all remaining. As it can be seen from Tables 1-4 the smaller values of $\lambda_{\max }$ (and thus weaker correlations) are quite systematically associated with the scaling exponents $\alpha$ which are larger than their average value $\langle\alpha\rangle=1.55$ while the larger values of $\lambda_{\max }$ (stronger correlations) are typically associated with $\alpha$ smaller than this average. For a better visualization the quality of such a tendency is demonstrated in Fig. 3 by the scatter plot of $\alpha$ versus the corresponding $\lambda_{\max }$ for all the 60 currencies. An overall decrease of $\alpha$ with increasing $\lambda_{\max }$ is quite evident from this Figure. It is faster in the region of smaller $\lambda_{\max }$ values and

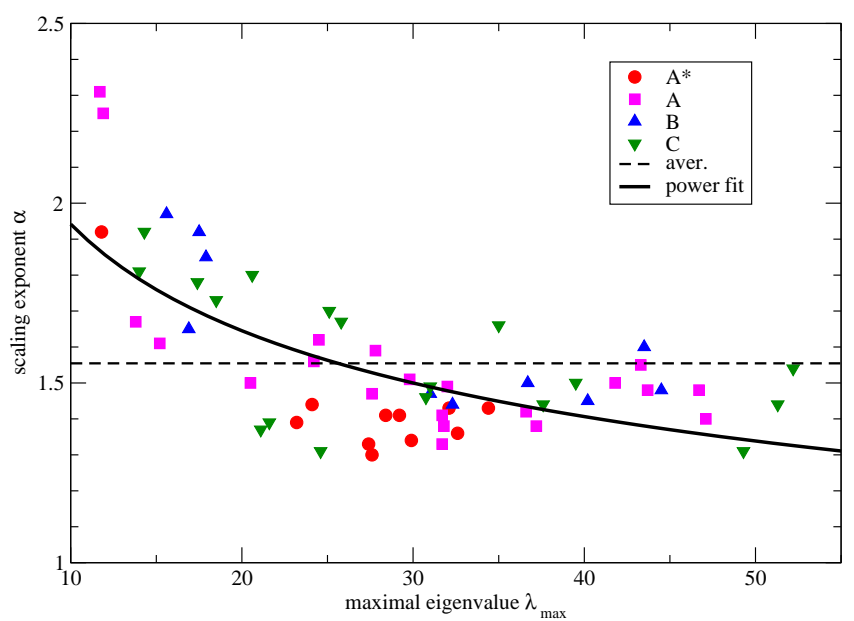

Fig. 3. Scatter plot of $\alpha$ versus $\lambda_{\max }$ for all the 60 currencies. Four different symbols correspond to four different groups of the currencies as described in the inset. The solid line represents the best fit in terms of a power function and the dashed horizontal line indicates the average $\langle\alpha\rangle$.

then tends to saturation. The best simple representation for this dependence (solid line in Fig. 3) is in terms of a power function $\left(\alpha \sim\left(\lambda_{\max }-\lambda_{\max }^{R M}\right)^{-\beta}\right)$ with $\beta \approx 0.21$. As $\lambda_{\max }^{R M}$ the upper bound of the Wishart ensamble of random matrices is used. Thus $\lambda_{\max }^{R M}=1+1 / Q+2 / \sqrt{Q}$ [14,15], where $Q=T / N$ which in our case gives $\lambda_{\max }^{R M}=1.413$.

The above correspondence between $\lambda_{\max }$ and $\alpha$ remains in accord and in fact constitutes an extension of the observation made in ref. [16 in connection with the stock market. There it is shown that the MST scaling exponent evaluated during crash periods - whose dynamics is inherently associated with stronger correlations [23] - is smaller than during normal business periods. As already mentioned and even more extensively discussed in [6] the currencies exchange rates expressed in terms of a less influential currency are more correlated as compared to the situation when the leading ones are used as reference. It is also interesting to see that the above coincidences apply to all the four groups of currencies listed in Tables 1-4 and displayed in Fig. 3 (where they are seen to overlap with each other) even though they are characterized by sizeably different liquidity and in some cases (group C) even by non-trade mechanism of setting the exchange rates. This implies that the general characteristics of the corresponding MST's are quite robust with respect to such factors.

The power like behavior with the scaling exponent $\alpha=1.6$, which is very close to the one estimated in the present study, was also found 13 for the stock market MST graphs. Furthermore, similar characteristics have been identified in several other natural and artificial complex networks studied so far in the literature - for references see [24,25] - and the world currency network considered here belongs to this universality class, with the scaling exponent in the range $1<\alpha<2$. 
A. Z. Górski et al.: Scale free effects in world currency exchange network

\section{References}

1. L. Laloux, P. Cizeau, J.-P. Bouchaud, M. Potter, Phys. Rev. Lett. 831467 (1999).

2. V. Plerou, P. Gopikrishnan, B. Rosenow, L. A. N. Amaral, H. E. Stanley, Phys. Rev. Lett. 831471 (1999).

3. J. Kwapień, S. Drożdż P. Oświęcimka, Physica A 359589 (2006).

4. Y. Aiba, N. Hatano, H. Takayasu, K. Marumo, T. Shimizu, Physica A 324253 (2003).

5. Sauder School of Business, Pacific Exchange Rate System, http://fx.sauder.ubc.ca/data.html (2006).

6. S. Drożdż, A. Z. Górski, J. Kwapień, Eur. Phys. J. B 58 499 (2007).

7. The algorithm to construct MST graphs was for the first time published by Czech mathematician, Otakar Borùvka in 1926.

8. J. Kruskal, Proc. Am. Math. Soc. 748 (1956).

9. C.H. Papadimitrou, K. Steigliz, Combinatorial Optimization, (Prentice-Hall, Englewood Cliffs 1982).

10. D.B. West, Introduction to Graph Theory (Prentice-Hall, Englewood Cliffs 1996).

11. R. N. Mantegna, Eur. Phys. J. B 11193 (1999).

12. J.-P. Onella, A. Chakraborti, K. Kaski, J. Kertész, Physica A 324247 (2003).

13. G. Bonanno, G. Caldarelli, F. Lillo, R. N. Mantegna, Phys. Rev. E 68 046130-1 (2003).

14. V. A. Marchenko, L. A. Pastur, Mat. Sb. 72507 (1967).

15. A.M. Sengupta, P.P. Mitra, Phys. Rev. E 603389 (1999).

16. J.-P. Onella, A. Chakraborti, K. Kaski, J. Kertész, A. Kanto, Phys. Rev. E 68 056110-1 (2003).

17. G. Bonanno, G. Caldarelli, F. Lillo, S. Miccichè, N. Vandewalle, R. N. Mantegna, Eur. Phys. J. B 38363 (2004).

18. M. McDonald, O. Suleman, S. Williams, S. Howison, N. F. Johnson, Phys. Rev. E 72046106 (2005).

19. A. Z. Górski, S. Drożdż, J. Kwapień, P. Oświęcimka, Acta Phys. Pol. B 372987 (2006).

20. M. J. Naylor, L. C. Rose, B. J. Moyle, Physica A 382199 (2007).

21. E. Ravasz, A.-L. Barabási, Phys. Rev. E 67026112 (2003).

22. J. D. Noh, Phys. Rev. E 67 045103(R) (2003).

23. S. Drożdż, F. Grümmer, A. Z. Górski, F. Ruf, J. Speth, Physica A 2874400 (2000).

24. R. Albert, A.-L. Barabási, Rev. Mod. Phys 7447 (2002).

25. S. Boccaletti, V. Latora, Y. Moreno, M. Chavez, D.-U. Hwang, Phys. Rep. 424175 (2006). 
